\title{
Zybride führen an die Grenzen der Leiblichkeit ... und darüber hinaus
}

\section{Abstract (English)}

In the field of genetics, new research dealing with the amalgamation of human and animal cells has been conducted over the last decade. This article discusses the case of inserting human DNA into the ovum of an animal due to a shortage of human eggs. The resulting so-called cybrid (cytoplasmic hybrid), produced for obtaining stem cells, is destroyed after 14 days. The article asks how we should react as theologians, ethicists and theological ethicists, when facing this new challenge. Fear of the bodiliness of this human-animal-embryo is a possible response to this experiment. Resulting issues concerning the cybrid are discussed, such as the fictional resurrection of the body, the non-availability of a body, and the problematic case of human dignity. Is the cybrid human or not and in what percentage? This question implies an ongoing discussion and has consequences for dealing with and reacting to this product, as well as the subsequent question of whether it might be more adequate to talk of a cybrid's human soul.

\begin{abstract}
Français)
Dans le domaine de la génétique, de nouvelles recherches, touchant à la fusion de cellules humaines et animales ont été menées ces dix dernières années. Cet article examine le cas de l'insertion d'ADN humain dans l'ovule d'un animal à cause d'un manque d'œufs humains. Le résultat, appelé cybride (hybride cytoplasmique), produit pour obtenir des cellules souches, est détruit au bout de 14 jours. L'article se demande comment, face à ce nouveau défi, nous devrions réagir en tant que théologiens, éthiciens et théologiens moralistes. Une réponse possible à cette expérience est la crainte quant à la corporéité de cet embryon humain-animal. Concernant ce cybride, on discute de problèmes comme la
\end{abstract}


résurrection (imaginée) du corps, la non-disponibilité d'un corps et le cas problématique de la dignité humaine. Le cybride est-il un humain ou non - et dans quel pourcentage? Cette question implique un débat et a des conséquences sur la manière de traiter et de réagir à ce produit. Une question subséquente est celle de savoir s'il serait plus adéquat de parler de l'âme humaine d'un cybride.

Über den menschlichen Körper und seine Einzelteile wird im Zeitalter der technischen Reproduzierbarkeit immer stärker verfügt, sei es indem Körpersubstanzen und -teile ersetzt werden (vgl. tissue engineering), sei es dass der Körper durch zahllose technische Maßnahmen verändert wird (vgl. Schönheitsoperationen). Die Posthumanisten wollen gar die menschliche Lebensform durch künstliche Intelligenz ergänzen. Ihrer Ansicht nach soll der Körper als Ort der Natur verlassen und zumindest teilweise in einen Ort der Technik und der Informationen umgebildet werden ${ }^{1}$. Eines gemeinsam haben all diese Entwicklungen: sie führen im Modus des Verfügens über den Menschen an die Grenzen ... seines Körpers, seines Leibes ${ }^{2}$ und seiner Seele. Einen Spezialfall bilden die Chimären- und Hybridbildung, welche die (Gattungs-)Grenzen der Leiblichkeit verwischen und die Frage nach der menschlichen Seele eines Zybrids, eines Produkts aus menschlicher DNA und tierischer Eizelle, exemplarisch aufwerfen. Mein grundsätzlicher Ansatz in diesem Beitrag lautet deshalb, wie dabei auftretenden Grenzüberschreitungen theologisch, ethisch und theologisch-ethisch begegnet werden kann.

\section{Chimären-, Hybridbildung und Zybride}

„Bei einer Chimäre besteht ein Embryo ... aus zwei Zelltypen mit unterschiedlichen Genomen. ${ }^{c 3}$ Naturwissenschaftlich betrachtet werden Lebewesen mit Genen oder Erbgut von mindestens zwei verschiedenen Arten als Chimären

1 Vgl. J. Manemann, Das Verschwinden des Körpers: Transhumanistische und posthumanistische Visionen, in ZME 54 (2008) 35-48, S. 37.

2 Die Begriffe Körper und Leib werden im 2. Punkt unterschieden.

3 M. ВоввеRт, Was macht Menschsein aus, wenn Biotechniken die Spezies verändern? Ethische Fragen der Forschung mit embryonalen Stammzellen, alternativen Klonverfahren und Chimären, in Ethica 15 (2007) 7-49, S. 30. Zur Problematisierung: „Chimären im Zusammenhang mit humanen embryonalen Stammzellen werden häufig so charakterisiert, dass ein Embryo aus zwei genetisch unterschiedlichen Zellpopulationen zweier oder mehrerer Spezies besteht. Dabei wird angenommen, dass sich diese auf genetischer Ebene nicht so vermischen, dass neue Keimzellen entstehen könnten - eben anders als bei Hybriden. Dass bei der Erzeugung von Chimären keine Genomvermischung stattfindet, lässt sich jedoch nicht ausschließen..." Bов Bert, Menschsein, S. 35. 
bezeichnet. Der Begriff kommt vom Griechischen „chimaira“ für Ziege und bezeichnet in der griechischen Mythologie ein Feuer speiendes Ungeheuer, „das vorn wie ein Löwe, hinten wie ein Drache und in der Mitte wie eine Ziege ${ }^{\text {"4 }}$ aussah. Eine Chimäre ist allgemein gesprochen ein Mischwesen aus Mensch und Tier.

Hybride dagegen sind Wesen, „die von der ersten Zelle an aus verschiedenen Genomen ${ }^{\text {c } 6}$ bestehen. Sie entstehen auf natürliche Weise z.B. bei der Verschmelzung der Keimzellen von zwei verschiedenen Arten oder Zuchtlinien (z.B. Maultier) oder auf künstlichem Wege (z.B. Hybridsaatgut). Sie sind also nicht Organismen, die aus genetisch verschieden zusammengesetzten Zellen bestehen (Chimären), sondern in ihnen ist jede einzelne Zelle genetisch gemischt zusammengesetzt und der gesamte Organismus hat in jeder Zelle die gleiche genetische Grundstruktur.

Als neueste "Errungenschaft" in diesem Bereich seien die Zybride (kurz für zytoplasmische Hybride/cytoplasmic hybrids) vorgestellt, die aus menschlicher DNA bestehen, welche aber in eine entkernte tierische Eizelle eingebracht wird?' da menschliche Eizellen nicht in ausreichendem Maße vorhanden sind. Das Erbmaterial eines so erzeugten Embryos stammt nicht vollständig vom Menschen, sondern zu einem - wenn auch überaus geringen - Prozentsatz auch von demjenigen Tier, dessen Eizelle verwendet wurde. Anfang September 2007 hat die britische Human Fertilisation and Embryology Authority (HFEA) in London die Züchtung von solchen Embryonen zu Forschungszwecken grundsätzlich erlaubt ${ }^{9}$. Ziel ist die Gewinnung menschlicher embryonaler Stammzellen ${ }^{10}$, wobei die entstehenden Zybriden nach 14 Tagen von den beantragenden

4 H.J. Rose, Griechische Mythologie: Ein Handbuch, München, Beck, 1955, S. 274.

5 Oder weiter gefasst: „Chimären sind Lebewesen, die fremde Organe oder fremde Zellen in sich tragen." M. BECK, Mensch-Tier-Wesen: Zur ethischen Problematik von Hybriden, Chimären und Parthenoten, Paderborn - München - Wien - Zürich, Schöningh, 2009, S. 21. Das Feld der Xenotransplantation wird in diesem Artikel ausgeblendet.

6 BECK, Mensch-Tier-Wesen, S. 87.

7 Es handelt sich um den somatischen Zellkerntransfer, das Klonen nach der "Dolly-Metho-

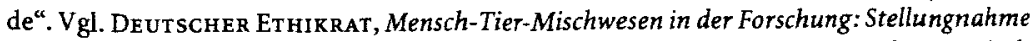
(2011), URL: http://www.ethikrat.org/dateien/pdf/stellungnahme-mensch-tier-misch wesen-in-der-Forschung.pdf [29.11.2010], S. 12.

8 The Human Fertilisation and Embryology Authority (HFEA) is a body set up by an Act of Parliament, the Human Fertilisation and Embryology (HFE) Act 1990, to oversee IVF treatment and research using human embryos in the UK.

$9 \mathrm{Vgl}$. M. HoffMANN, Interspezies-Embryonen: Die "neue Sprache" der Forschung und die Sprachlosigkeit der Moraltheologie?, in ZME 54 (2008) 333-346, S. 335.

10 "Langfristig sollen Zytoplasmatische Hybride die Herstellung von patientenspezifischen pluripotenten Stammzellen ermöglichen, aus denen Zellen zur Therapiezwecken gezüchtet werden können, die genetisch weitgehend mit den Zellen des Patienten identisch sind." Deutscher Ethix Rat, Mensch-Tier-Mischwesen, S. 16. 
Forschergruppen ${ }^{11}$ wieder zerstört werden ${ }^{12}$. In China und den USA werden Kaninchen- oder Kuh-Eizellen dafür verwendet.

Dieses neue Verfahren hat bereits Aufsehen in Kreisen der katholischen Kirche erregt ${ }^{13}$. Die moralische und sittliche Unannehmbarkeit (vgl. Dignitas Personae, 33) scheint zwar für das kirchliche Lehramt auf der Hand zu liegen. Es bleibt jedoch die Aufgabe für die theologische Ethikerin, die dabei im Hintergrund stehenden Argumente kritisch zu beleuchten. Dies soll vor allem unter dem Gesichtspunkt der (bis jetzt zumeist virtuellen) Körperlichkeit solcher Zybride geschehen.

In einem ersten Punkt dieses Beitrags wird unterstrichen, wie wichtig die Leiblichkeit im Gebäude des katholischen Glaubens ist (hier sei nur das Stichwort der Auferstehung des Leibes genannt), in einem zweiten Punkt wird die Unverfügbarkeit des Leibes diskutiert und drittens die mögliche Menschenwürde eines Zybrids unter die Lupe genommen. Besonders spannungsvoll ist diese Betrachtung dadurch, dass die vorgestellten Zybride nach 14 Tagen zerstört werden. Dem hier analysierten Produkt wird bis jetzt kein phänotypisch ausgebildeter Körper zugestanden, da sie rein zu Forschungszwecken in-vitro hergestellt werden.

\section{Der Herausforderung begegnen}

1. Als Theologin

Im Apostolischen Glaubensbekenntnis heißt es „Ich glaube an die Auferstehung des Fleisches", nicht nur an die Auferstehung. Dieser Satz gehört seit frühester

11 Die ersten Erfolge wurden berichtet: http://news.bbc.co.uk/2/hi/health/7323298.htm [29, 11 . 2010].

Der Deutsche Ethikrat kommt zu einer zurückhaltenden Einschätzung: ${ }_{n}$ Zusammenfassend kann man somit sagen, dass die Forschung an Zytoplasmatischen Hybriden bisher hinter den Erwartungen zurückbleibt. Allerdings ist es aufgrund der noch schmalen Datenbasis verfrüht, das Potenzial der Technik abschließend zu bewerten." (Deutscher Ethikrat, Mensch-Tier-Mischwesen, S. 16).

12 „Es ist offensichtlich nicht ganz klar, wie weit diese Mensch-Tier Hybride sich entwickeln könnten. Sie sind in den erwähnten Studien bis zum Blastocystenstadium entwickelt worden, aber dann wurden die Versuche abgebrochen, da es möglich schien, dass diese Wesen sich auch implantieren und weiter entwickeln könnten. “ BECK, Mensch-Tier-Wesen, S. 252.

13 Vgl. Kongregation fūr die Glaubenslehre, Instruktion Dignitas Petsonae über einige Fragen der Bioethik (VAS, 183), Bonn, Seluretariat der Deutschen Bischofskonferenz, 2008, S. 33. 
Zeit zu den Grundbeständen des christlichen Glaubens ${ }^{14}$. So zum Beispiel Tertullian: Fiducia Christianorum resurrectio mortuorum. Illam credentes sumus ${ }^{15}$.

Der Hauptgrund, warum die Christen trotz aller Widerstände am Glauben an die Auferstehung des Fleisches festhalten, ist die Auferstehung Christi (Apg 26,36, vgl. 1 Kor 15,20-23). „Christus ist nicht alleine auferstanden. Seine Auferstehung ist der Anfang, die Ursache, ja in gewisser Weise schon die Realisierung der allgemeinen Auferstehung von den Toten. ${ }^{\text {"16 }}$ Die Lehre der katholischen Kirche sagt, am Ende der Zeiten werden alle Menschen „mit dem eigenen Leib, den sie hier tragen, auferstehen ${ }^{\text {"17 }}$. Dies wird deutlich an Jesu Auferstehung: „der Auferstehungsleib ist real identisch mit dem irdischen Leib. Die Evangelienberichte betonen diese Identität ganz ausdrücklich ${ }^{\text {“18 }}$; er ist aber auch davon unterschieden, z. B. als Jesus von den Jüngern nicht sofort wiedererkannt wird.

Mit diesen kurzen Skizzen sei an die Wichtigkeit des Leibes für die Christen erinnert. Bezüglich des Zybrids sind verschiedene spannungsvolle Fragen für dessen Leib aufgeworfen: Kann der Zybrid, sofern er glauben kann, auf die Auferstehung des Leibes hoffen? Und welcher Leib wird auferstehen? Natürlich handelt es sich bei diesen Fragen um Gedankenkonstruktionen aus dem Bereich der Science Fiction, die aber durch solche humangenetische Experimente initiiert werden. Über diese rein hypothetischen Fragestellungen hinaus bleibt die Bedeutung des Leibes und des Körpers für den christlichen Glauben bestehen.

\section{Als Ethikerin}

kann man dem Diskurs um die Leiblichkeit mit der Position der Unverfügbarkeit des menschlichen Körpers begegnen: Weder man selbst noch andere dürfen über den Körper verfügen, denn die Selbstannahme ist die Voraussetzung für eine sinnvolle Sorge um den Leib ${ }^{19}$. Im Ansatz, die Unverfügbarkeit des Körpers hochzuhalten, wird von einem weiten Sinn des Verfügens ausgegangen (Wie-

14 Seit der Liturgiereform: „Auferstehung der Toten“. Zur Genese vgl. C. SCHöNBorn, „Auferstehung des Fleisches" im Glauben der Kirche, in IKaZ Communio 19 (1990) $13-29$.

15 Tertullian, Opera montanistica (CCSL, 2), Turnhout, Brepols, 1954, S. 921.

16 SchönboRn, Auferstehung, S. 19.

17 Glaubensbekenntnis des IV. Laterankonzils (1215) (DH 801).

18 SchönboRn, Auferstehung, S. 23.

19 Verfügen muss hierbei in zweifacher Weise verstanden werden. In einem weiten Sinn sind damit alle Maßnahmen gemeint, die den Körper wiederherstellen (unter den Sinnzielen des ärztlichen Handelns). In einem engen Sinn von Verfügen geht es um die Herstellung. Vgl. G. Pölt Ner, Sorge um den Leib - Verfügen über den Körper, in ZME 54 (2008) 3-11, S. 4. Vgl. auch seine Neuerscheinung G. PöLTNER, Leib und Leben: Bioethische Erkundungen zur Leiblichkeit des Menschen, Göttingen, Vandenhoeck \& Ruprecht, 2010. 
derherstellung), d.h. bestimmte Eingriffe sind erlaubt, aber grundsätzlich wird die Integrität des Körpers angenommen und versucht zu bewahren.

Dieser Ansatz steht dem naturalistischen und materialistischen gegenüber, der den Körper als Instrument betrachtet und damit jedweder Vermischung von Mensch und Tier positiv gegenübersteht, so lange sie den menschlichen Körper optimiert.

„Das Verfügenwollen über den eigenen Körper (im engen Sinn von, verfügen') basiert auf einem heimlichen Dualismus. Der Mensch wird als Subjekt angesetzt, das sich seines Körpers bedient. Der Dualismus wird durch die euphorische Präsentation von Optimierungsprogrammen ebenso verdeckt, wie die mit ihm verbundene heimliche Abwertung des Leibes. Der Leib ist nicht gut genug. ${ }^{\text {"20 }}$

Diese instrumentelle Betrachtungsweise bricht mit der Einsicht, dass die Selbstannahme die Voraussetzung für ein sinnvolles Sorgen um den eigenen Körper ist. Eine philosophische Strebensethik würde dem entgegnen, dass der Umgang mit dem Eigenen für ein gutes und gelingendes Leben wichtig ist: Es sei hier nur auf das Erleben des eigenen Körpers und das damit verbundene Erleben der eigenen Grenzen hingewiesen ${ }^{21}$.

Außerdem sei als Ethikerin an die Differenz zwischen Körper und Leib gedacht. Der Mensch hat nicht nur Körper, sondern ist auch Leib.

„Der Begriff des Körpers bezeichnet die Möglichkeit der Vergegenständlichung, während ,Leib' die Durchformung des Körpers zum Ausdruck bringt, den lebendigen Körper, der Selbst- und Weltbezogenheit beinhaltet. ${ }^{\star 22}$

Körper haben und Leib sein müssen als Einheit in Differenz gedacht werden, um den Menschen ganzheitlich zu betrachten. Der je spezifische Leib verbürgt die Individualität des Menschen ${ }^{23}$, wobei der Mensch mehr ist als (nur) sein Leib, denn durch ihn ist der Mensch in der Welt verortet: Er ist zugleich Grenze und Brücke zwischen dem Innen und Außen der Umwelt.

Die Unverfügbarkeit des menschlichen Körpers und des Leibes ist besonders bei den Zybriden neu durchzubuchstabieren. Das Töten nach 14 Tagen, nachdem daraus pluripotente embryonale Stammzellen gewonnen wurden, findet ihre Begründung u.a. in jener zu Beginn beschriebenen materialistischen und naturalistischen Sichtweise auf den Körper, die die Zybride nur als Instrumente

20 Pöltner, Sorge, S. 10.

21 Vgl. U. MÜLLER, Zum Verhältnis von Körperlichkeit und Unverfügbarkeit, in ZME 54 (2008) $73-80,5.77-78$.

22 Manemann, Verschwinden, S. 43.

23 mIch bin Leib - dieser Leib bin ich." Das ist nicht der manipulierte Leib mit Body-Building und Face-Lifting, das ist meine individuelle Gestalt und Lebensgeschichte." E. MoltmanNWendel - J. MoltmanN, Mit allen Sinnen glauben: Überlegungen zur Auferstehung des Fleisches, in StZ 223 (2005) 723-735, S. 725. 
ansieht. Da zu wenige menschliche Eizellen zu Forschungszwecken vorhanden sind (die Gewinnung ist für die betroffenen Frauen körperlich und psychisch belastend ${ }^{24}$, werden die Gattungsgrenzen durchbrochen und stattdessen tierische Eizellen verwendet. Damit sind die Zybride vollständig für die Forschung instrumentalisiert.

\section{Als theologische Ethikerin}

beschäftigt in besonderer Weise die Frage nach der Menschenwürde. Zybride „können aufgrund ihres Genoms nicht mehr vollständig der menschlichen Rasse zugeordnet werden ${ }^{625}$. Haben diese damit keine Menschenwürde?

Diese Frage kann scheinbar nur mit einem genauen Blick in die genetischen Informationen solcher Zybride beantwortet werden: $0,1 \%$ des genetisches Materials stammen vom Tier, der Rest von der genetischen menschlichen Kerninformation. Entscheidet das genetische Material über die Artzugehörigkeit: Sind die Zybride mehr Mensch oder mehr Tier? Ist die weibliche Eizelle der Kuh oder des Kaninchens nur ein Mittel der Reprogrammierung des menschlichen Zellkerns oder prägt diese Eizelle der Kuh/des Kaninchens diesem entstehenden Wesen auch ihr "Kuhsein/Kaninchenseins" $\operatorname{ein}^{26}$ ? Die in der Eizelle vorhandene 0,1 Prozent mitochondriale DNA scheint keine zentrale Rolle zu spielen, wenngleich sie in jeder einzelnen Zelle dieses neuen Organismus (Energiestoffwechsel) vorhanden ist.

„Nach der hier auftretenden Auffassung befinder sich dieser menschliche Zellkern, der durch eine tierische Eizelle reprogrammiert wird, in einer menschlichen Embryonalentwicklung. Er ist ein menschlicher Embryo ... Ein wirkliches Mischwesen entstünde dann, wenn man Spermien vom Menschen und eine Eizelle von der Kuh (Kaninchen) zusammenbrächte. ${ }^{« 27}$

Diese Einschätzung von Prof. Matthias Beck (Wien) wird nicht immer geteilt (vgl. Prof. Dr. Jens Reich, Mitglied des Deutschen Ethikrates) ${ }^{28}$.

24 "Oocyte donation is a very demanding physical process, with its hormonal induction treatment causing manifestations of ovarian hyper stimulation syndrome in up to one third of treated women. Severe forms requiring hospitalisation and potentially life-threatening are less common but by no means rare." Vgl. S. CAMPoREsI - G. Boniolo, Fearing a nonexisting Minotaur? The ethical challenges of research on cytoplasmic hybrid embryos, in Journal of Medical Ethics 34 (2008) 821-825, S. 825. Die Kommerzialisierung des menschlichen Körpers und die Ausbeutung der Frauen seien hier noch ergänzt.

25 Vgl. Hof PMANN, Interspezies-Embryonen, S. 340-345.

26 Vgl. BECK, Mensch-Tier-Wesen, S. 88.

27 Ibid, S. 252 f. Beck bezieht sich hier auf Rudolf Jaenisch.

28 ,Ich halte sie nicht für vernachlässigbar, nicht in dem Sinne, wie wir ja sonst auch alle möglichen tierischen Gene und Zellen durch die Nahrung und bei anderen Gelegenheiten 
Im Grunde geht es um die Bedeutung der mitochondrialen DNA: Ist sie qualitativ vernachlässigbar oder nicht? Im ersten Fall ist das Ergebnis ein menschlicher Embryo, der mit allen Argumenten pro und contra im Zusammenhang mit dem biomedizinischen Forschungsklonen behandelt werden kann. Ist man aber der Meinung, die mitochondriale DNA sei nicht zu vernachlässigen (so seien in jeder Zelle nun Kuh- oder Kaninchengene vorhanden, die Mitochondrien fungieren als Träger von genetischen Defekten usw.), so ist dieses Wesen doch eher ein Mensch-Tier-Mischwesen mit eigener genetischer Identität. Die Frage nach der Artgrenze stellt sich doch sehr deutlich und verlangt nach einer eigenen ethischen Beurteilung ${ }^{29}$. Mit dieser Auswertung der genetischen Zusammensetzung von Zybriden ist - abschließend gesagt - keine weitere Klärung erfolgt, da bereits auf der biologischen Ebene (noch) Unsicherheiten bestehen.

Ein Ausflug in die Geschichte der Menschenwürde problematisiert des Weiteren diese einfache Deduktion des Menschseins aus den Genen. Lange Zeit war es unumstritten, dass der Grund der Menschenwürde in jener spezifischen Differenz zu suchen ist, die den Menschen einerseits als Person auszeichnet und andererseits vom Tier differenziert. Beide Grenzen werden immer mehr aufgeweicht, z. B. die Grenzen zu den Tieren bei den Zybriden.

Im Grundsatz liegt das Problem darin, ob die Menschenwürde als Vorgabe oder Zuschreibung angesehen wird. In der ersten Position: Menschenwürde fundiert in der Vorgabe der wesentlichen Seinsweise des Menschen und kann nicht von jemand anders ab- oder zugesprochen werden. Für den Zybrid hat dies folgende Konsequenz: Jeder von ihnen hat Menschenwürde ... mit wie viel menschlichen und tierischen Zellen auch immer, wenn er als menschlicher Embryo eingestuft wird. Die Menschenwürde stellt ein „Wesensmerkmal dar, welches allen Menschen allein aufgrund ihres Menschseins gleichermaßen zukommt - also unabhängig von Leistung, Amt oder Ansehen sowie unabhängig von Geschlecht, Alter, Rasse oder Religion. Würde besagt demnach die prinzipielle Gleichheit oder Gleichwertigkeit aller Menschen, ohne deshalb die Differenz und Andersheit von Individuen zu nivellieren ${ }^{\star 30}$.

aufnehmen und auch andere Organismen in unserem Körper haben. Ich weiß noch nicht, wie ich es beurteilen würde, wenn alle unsere Zellen lebenswichtige Rinder-Gene enthalten. Was wir alle in uns tragen, sind jedoch lebenswichtige Gene, die eigentlich von Bakterien abstammen. Vernachlässigbar oder unerheblich, eine Banalität oder eine Bagatelle oder so etwas ist das jedenfalls nicht." Wortprotokoll der öffentlichen Sitzung des Deutschen Ethikrates am 26.6.2008, vgl. DeUT SCHER ExHI RRAT, Wortprotokoll: Niederschrift über den offentlichen Teil der Plenarsitzung des Deutschen Ethikrates am 26. Juni 2008 in Berlin, URL: http://www.ethikrat.org/dateien/pdf/Wortprotokoll_2008-06-26_Website.pdfi

[29.11. $2010]$, S. 27.

29 Deutscher Ethikrat, Wortprotokoll, S. $32 \mathrm{f}$, Beitrag von Prof. Dr. Eberhard Schockenhoff.

30 G. MARSCHŨTz, theologisch - ethisch - nachdenken, vol. 1, Würzburg, Echter, 2009, S. 242. 
Wird die Menschenwürde als (zumeist erst nachträgliche) Zuschreibung verstanden (der zweite Fall), bestimmt eine andere Instanz, ob dieser Zybrid Menschenwürde besitzt, oder auch nicht. Vor allem da er nicht zu $100 \%$ menschliches Genom besitzt, ist es ein Leichtes, ihm die Menschenwürde abzusprechen und das Töten nach 14 Tagen als gegeben darzustellen.

Im britischen Raum sind solche Zybride in den Laboren hergestellt worden, so dass die Bischöfe von England und Wales eine Stellungnahme zu diesem Verfahren herausgaben: Neben der fehlenden Elternschaft ${ }^{31}$ und der "unnormalen" Natur der entstandenen Zellen, die mit noch nicht absehbaren Risiken (vgl. Übertragung tierischer Krankheitserreger oder mangelnde Qualität der Mitochondrien-DNA) ${ }^{32}$ verbunden sind, ist es vor allem die Menschenwürde, die in Zusammenarbeit mit dem Anscombe Bioethics Centre, ehemals Linacre Center for Healthcare Ethics, ins Feld geführt wird:

„Selbst wenn man die Gefahr vernachlässigt menschliche Embryonen zu erschaffen, indem man davon ausgeht, dass solche Embryonen nicht erzeugt würden, halten wir trotzdem daran fest, dass die Herstellung von Hybriden und Chimären gegen die Menschenwürde verstößt. ${ }^{\text {(33 }}$

Die Zybride verletzen die Menschenwürde. Näher bestimmt wird sie jedoch nicht.

Aber nicht nur die personale individuelle Würde jedes einzelnen Zybrids wird verletzt, sondern der Idee der Menschheit (Gattungswürde) und ihrem Charakteristikum, sich selbst Zwecke setzen zu können, wird laut Matthias Beck mit

31 Vgl. Catholic Bishops' Conference of England and Wales and Linacre Centre for HealthCARE ETHICs, Joint Response to the Human Tissue and Embryos (Draft) Bill (2007), URL: http://www.linacre.org/Linacre\%20Joint\%20submission\%20on\%20Human\%20Tissue $\% 20$ and $\% 20$ Embryos\%20_draft_\%20Bill.pdf [29.11.2010].

32 "It should be remembered that animal-human hybrids and chimeras are in any case likely to be of limited scientific value, due to the abnormal nature of their cells. They are unlikely to provide any treatments for patients, in view of the medical risks which they carry: risks not limited to the transmission of animal viruses to humanS. It should be remembered that mitochondrial problems are a key factor in many neurodegenerative diseases; there would also be particular risks if hybrid cells were used to treat heart or liver complaints, for which cloning has been proposed as a solution." Linacre Centre Submission to the Science and Technology Committee Inquiry into Government Proposals for the ReguLATION OF HYBRID AND CHIMERA EMBRYOS, URL: www.linacre.org/hybridschimeras.pdf [29.11.2010], S. 3. Vgl. auch BECK, Mensch-Tier-Wesen, S. 90. "Außerdem sind alle Klone krank ... Die Klone mit tierischen Eizellen sind noch mehr geschädigt, weil die Eizelle offensichtlich nicht das genügend große Potenzial zur Reprogrammierung hat."

33 "Leaving aside the risk of creating human embryos, and assuming that such embryos would not be created, we believe the production of hybrids and chimeras can still offend against human dignity. "Linacre Centre Submission to the Science and Technology Committee Inquiry into Government Proposals for the Regulation of Hybrid and Chimera Embryos, S. 3. 
diesem Verfahren widersprochen. Mit den vorgestellten Zybriden werden Wesen hergestellt, bei denen es zu einer eigenen Zwecksetzung gar nicht komme ${ }^{34}$.

Wie in diesem Punkt deutlich geworden, ist das Kriterium der Gattungszugehörigkeit ${ }^{35}$ immer wichtiger für die Frage nach der Menschenwürde ${ }^{36}$. Und es wird sichtbar, dass auch der Begriff der Menschenwürde selbst durch solche neue Formen des Lebens problematisiert wird. Wird die Menschenwürde nun als Vorgabe oder als Zuschreibung verstanden: In beiden Fällen ist die Menschenwürde für den Zybrid nicht gesichert, da der Einfluss der tierischen Eizelle nicht geklärt ist. Es stellt sich im Grundsätzlichen die Frage, ob die Menschenwürde als absoluter Wert überhaupt noch in den neuen bioethischen Forschungsfeldern tragfähig ist.

\section{Synthese: An die Grenzen der Leiblichkeit und darüber hinaus - Haben Zybride eine menschliche Seele?}

Die Erzeugung von Zybriden führt an die Grenzen der Leiblichkeit. Mit der Möglichkeit der Austragung eines Zybrids im weiblichen Körper werden Ängste geschürt. Zur Beruhigung wird von den ausführenden Wissenschaftlerinnen und Wissenschaftlern angeführt: Es bleibe bei einem bloß 14 Tage alten Wesen, im experimentellen Kontext der Grundlagenforschung geschaffen ${ }^{37}$. Ein Einsetzen in die weibliche Gebärmutter sei ein kriminelles Verbrechen ${ }^{38}$.

Aber führt man dieses Experiment zu Ende, kann man verschiedene Nachteile für die entstehenden Wesen sehen, z.B. dass ihnen die Findung eines Selbst-

34 Vgl. M. BECK, Hybride - Chimären: Mensch-Tier-Mischwesen: Ethische Fragen, URL: http:// www.ethikrat.org/dateien/pdf/beck_hybride-chimaeren.pdf [29.11.2010].

35 „Obwohl die Beeinträchtigung der Gattungswürde ein schwächeres Argument als eine direkte Verletzung der individuellen Menschenwürde darstellt, kann diese berürt sein, wenn die Identität und Eindeutigkeit der Gattung als solche bedroht sind. "DEUTSCHER ETHIKRAT, Mensch-Tier-Mischwesen, S. 37.

36 „Wenn die Spezies moralisch schützenswert ist, dann könnte dies zur Vorsicht Anlass geben, also zur Forderung führen, dass die Artgrenzen des Homo sapiens nicht verletzt werden dürfen. Man kann allerdings auch argumentieren, dass - sobald Chimären bestehen - diese unter dem Kriterium der Gattungszugehörigkeit nicht mehr schützenswert sind. ${ }^{\text {G }}$. BADURA-LOTTER - M. DÜWELL, Chimären und Hybride: Ethische Aspekte, in Jahrbuch für Recht und Ethik 15 (2007) 83-104, S. 89. Wobei das Kriterium der Gattungszugehörigkeit selbst wieder problematisiert werden muss.

37 Von der HFEA als human admixed embryo bezeichnet.

$38,4.10 \ldots$ No scientist has ever expressed an interest in transferring such embryos in the hope that a baby, if that were medically possible, would develop. Even if they did, it would be a criminal offence to do so." HFEA, Hybrids and Chimeras: A consultation on the ethical and social implications of creating human/animal embryos in research (2007), URL: http://www. hfea.gov.uk/docs/Hybrids_Chimera_review.pdf [29.11.2010], S. 15. 
verständnisses und einer biologischen Identität erheblich erschwert wäre. Soll der Zybrid sich als Mensch oder Tier verstehen? Beide Gruppen sind wahrscheinlich nicht bereit, den Zybrid in ihre Gruppe aufzunehmen ${ }^{39}$. Auch die gängigen Argumente gegen gentechnische Veränderungen bzw. bei zu Forschungszwecken erzeugten Embryonen (Eingriff in die Natur, Dammbruchargument, Risikoabwägung, Frage nach der möglichen Potenzialität usw.) könnte man noch anführen oder auch Fragen der Tierethik, aber als Theologin steht es doch an, nachdem die Leiblichkeit solcher Zybride problematisiert wurde, die damit einhergehende Frage ${ }^{40}$ nach der menschlichen Seele zu stellen, die wiederum nicht unumstritten ist.

Die katholische Lehre besagt, die Beseelung erfolge im Moment der Verschmelzung von Ei- und Samenzelle (Karyogamie) ${ }^{41}$. Geht man von diesem Fixpunkt aus, so gibt es keinen seelen-freien Leib. Dem oft dagegen angeführten Fall der eineiigen Zwillinge kann mit einem Verständnis der Individualität nicht als prinzipielle Unteilbarkeit, sondern als das „konkrete Ungeteiltsein einer organischen Ganzheit ${ }^{\text {c42 }}$ entgegnet werden.

Auch für den Zybrid stellt sich die Frage der Beseelung: Welche Rolle spielt die tierische Eizelle? Ist sie nur ein Trägermedium, in das der beseelte menschliche Embryo eingebracht wird? Oder ist die mitochondriale DNA in ihrer Funktion als späterer Energielieferant ein wesentliches Konstitutivum für die Erschaffung von etwas Neuem, noch Unbeseeltem und mit der Erschaffung dann beseeltem Etwas? Betrachtet man mit dem Theologen und Biologen Ulrich Lüke die Seele, insofern sie als von Gott geschaffen angesehen wird, als Ausdruck der Gottun-

$39 \mathrm{Vgl}$. K. GRöNER, Klonen, Hybrid- und Chimärenbildung unter Beteiligung totipotenter menschlicher Zellen, in H.-L. GÜNTHER - R. KELLER (Hgg.), Fortpflanzungsmedizin und Humangenetik: Strafrechtliche Schranken?, Tübingen, Mohr, 1987, 293-325, S. 303-304.

40 „Es wird eine Unterscheidung zwischen ,Körper‘ und ,Leiblichkeit" möglich, die Origenes mit seinen Gedanken der charakteristischen Gestalt schon gesucht hatte, aber mit seinen Denkmitteln noch nicht formulieren konnte. Nicht die einzelnen Atome und Moleküle als solche sind ,der Mensch' und nicht an ihnen hängt daher die Identität der ,Leiblichkeit'; sie hängt vielmehr daran, daß Materie unter die Ausdruckskraft der Seele tritt. So wie die Seele sich nun einerseits von Materie her definiert, so ist umgekehrt der Leib ganz von der Seele definiert: Leib, und zwar identischer Leib, ist das, was die Seele sich als ihren körperlichen Ausdruck baut. Gerade weil die Leiblichkeit nun so unlösbar zum Menschsein gehört, wird die Identität der Leiblichkeit nicht von der Materie, sondern von der Seele her bestimmt." I. RatZINGER, Eschatologie: Tod und ewiges Leben (Kleine Katholische Dogmatik, 9), Regensburg, Pustet, 1977, S. 148.

41 Dies wird nicht als singulärer Schöpfungsakt Gottes verstanden, sondern als Aktion Gottes und als Reaktion des Menschen, nach dem Modell von Wort und Antwort. Dann erst kann von Kommunikation gesprochen werden, in diesem Fall zwischen Gott und Mensch, wie der Theologe und Biologe Ulrich Lüke ausführt. Vgl. U. LükE, Das Säugetier von Gottes Gnaden: Evolution, Bewusstsein, Freiheit, Freiburg - Basel - Wien, Herder, ${ }^{2} 2006$, S. 151.

42 Lüke, Säugetier, S. 161. 
mittelbarkeit eines jeden Menschen ${ }^{43}$, so spielen die 0,1\% keine Rolle mehr, denn damit ist die Seele Chiffre für den Geschenkcharakter jeder menschlichen Existenz, egal in welcher genauen genetischen Zusammensetzung.

In einem ersten Schritt hat die Frage nach der Leiblichkeit die Problemfelder, die mit der Zybridbildung einhergehen, aufgerissen, auf den Punkt gebracht wurde die Problematik aber bei der Frage nach der Beseelung. Gestaltet sich die Frage nach der Leiblichkeit und dem sich später ausbildenden Körper schon schwierig, so ist die Frage nach der Seele nicht einfacher zu beantworten. Die Bedeutung der Leiblichkeit und der leib-seelischen Einheit veranschaulichen die komplexen Problemstellungen, die sich aus neueren Forschungsvorhaben ergeben, die rein auf naturwissenschaftlicher Ebene betrachtet, mit der Ersetzung der menschlichen durch die tierische Eizelle die ethischen Probleme der Ejzellgewinnung als auch, wenn man von einem Mensch-Tier-Wesen ausgeht, die Diskussion um den moralischen Status des Embryos und der Embryonenforschung umgeht bzw. löst. Aber gerade die Verwendung der tierischen Eizelle führt zu neuen und neuartigen Problemen, die das Menschsein auf substantielle Weise betreffen. 